\title{
Designing for a Bigger Picture: Towards a Macrosyntax for Information Visualizations
}

\author{
Florian Windhager, Günther Schreder, and Eva Mayr
}

\begin{abstract}
Information visualizations can amplify human cognition by transferring strenuous cognitive operations with abstract data into visual reasoning processes with external graphic representations. Cognitive scientists have conceptualized the internal representations emerging in such distributed cognitive systems as mental models, whose structures and dynamics are modeled on the basis of the external representations. While visual-syntactical rules for the construction of simple representations are well defined by different visualization methods and can be relatively easily internalized by the user, an essential question remains how to synthesize coherent macromodels from multiple views. To address this question from a mental models perspective, we assemble and discuss visual coherence techniques, which assist users in assembling "bigger pictures" of complex, abstract subject matters. As such we contribute to a macrosyntax for information visualizations, to more systematically support macrocognitive synthesis and reasoning operations. We delineate and exemplify three different strategies of coherence techniques: methods to support the initial construction of mental models, methods to support the sequential integration of information, and methods to support the synchronous integration of local insights into a global representation.
\end{abstract}

Index Terms-Mental model, model-based reasoning, distributed cognition, theory, information visualization, macrocognition, coherence techniques

\section{INTRODUCTION}

When reflecting on its purpose, information visualization (InfoVis) is used to take a functional stance: It is the amplification and augmentation of cognition in face of the challenges posed by abstract data, which is providing its practical justification [48] [6]. Accordingly, numerous authors investigated the cognitive effects of InfoVis tool use - from insights theory [39] to sensemaking [43] and reflected on relevant implications for InfoVis methods development and design from a cognitive science perspective [15] [30]. Yet looking at the state of research, relatively little is known about how users assemble local perceptions or projections of data into bigger pictures, in terms of globally integrated representations. To move towards a more comprehensible and interoperable design of complex InfoVis interfaces, this paper investigates how users build up more complex mental representations of bigger data sets and how design techniques can support these processes.

To take first steps in this direction, we build on the theory of mental models (MMs) - as it has been proposed by frameworks of distributed cognition and model-based reasoning [30] [31] - and discuss previously unregarded implications for a macrocognitive level of operations. With the term macrocognition we refer to cognitive operations and challenges emerging from sensemaking with more complex data and tasks [24] [26]. Dealing with complex InfoVis tools - often implemented as systems with multiple views (MVs) - users have to connect multiple perspectives and assemble local insights into bigger pictures. The results of such macrocognitive syntheses can vary greatly with regard to their global coherence and consistency - and hence support or even impede various local or microcognitive sensemaking processes via their topdown effects on perception and interpretation. Following a distinction by Tversky [56] we will reserve the concept of mental models for higher quality (i.e. coherent and consistent) syntheses only. In the following, we investigate various design methods how to

- Florian Windhager, Günther Schreder, and Eva Mayr are with the Department of Knowledge and Communication Management,

Danube University Krems, Austria.

E-mail: \{firstname.lastname\}@donau-uni.ac.at

Web: https://donau-uni.ac.at/florian.windhager

Manuscript, 2017 better support their coherent construction and elaboration. We will refer to these macro level design methods as coherence techniques and distinguish three different strategies: Methods supporting the initial construction of mental models, methods supporting the sequential integration of multiple views, and methods supporting the parallel integration of multiple views.

By looking at these coherence techniques in the context of a model-based reasoning framework we aim to initiate a more systematic discussion and implementation of such techniques. As their orchestrated development and evaluation tends to get lost in between the work on individual methods or views, we hope to establish the support of macrocognition as a higher level design task of its own. As a byproduct, we want to draw together theoretical threads and perspectives on the complex interplay of cognition and visualization, which have not seen a conceptual macro-synthesis up to now.

The following chapter reconstructs the theoretical discussion on mental models and distributed cognition with specific regard to the use of InfoVis methods and tools and moves them to a macrocognitive level. On this basis, the third chapter focuses on contributions to a macrosyntax for InfoVis and assembles and organizes visual coherence techniques, which can support InfoVis systems design and their users on a macrocognitive level. To guide more systematic research on this macrosyntax in the future, we present appropriate evaluation scenarios and techniques in chapter 4. The outlook finally discusses implications for InfoVis research and practice.

\section{Internal Representations between Cognitive Collages and Mental Models}

InfoVis research focuses on the development of visual tools as external means to support the construction and manipulation of internal representations of cognitive systems. By doing so, the limited skills of unaided human cognition (e.g. in face of abstract data and complex, invisible subject matters) take center stage, which are aided by InfoVis systems as "cognitive amplifiers", "cognitive mediators", or "cognitive prostheses" [2]. To do so effectively it seems obvious that InfoVis design should build on existing knowledge about mental representations and operations [31]. Yet current research into the workings of internal representations builds on different theoretical frameworks, producing a fair amount of terminological and theoretical diversity - which becomes also visible 


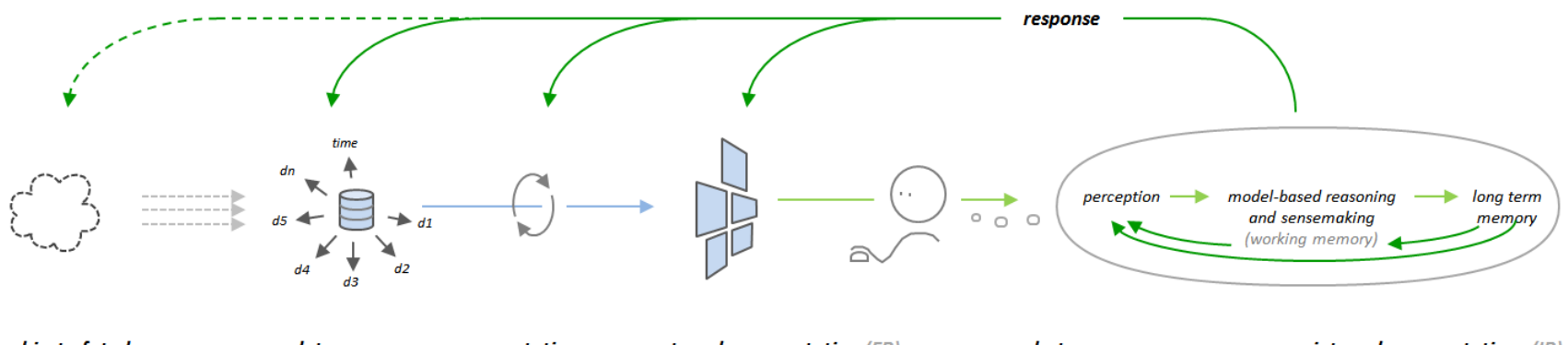

object of study

data

computation

external representation (ER)

analyst

internal representations (IR)

Fig. 1. Schematic line up of an extended cognition system, showing the translation from complex data and objects of study (left) to an external representation system (blue), which is (re)constructed as an internal representation by a reasoning and sensemaking system in working memory, and stored in long term memory (right). Cognitive bottom up-processes in bright, top down-processes in dark green.

throughout discussions about InfoVis foundations [44]. Conceptual diversity and controversies can even be found within specific theoretical domains, like with the theory of distributed cognition and the theory of mental models, on which we build the following considerations. To make our theoretical choices transparent, we outline conceptual origins and subsequent adaptations, to introduce a framework extension covering the macrocognitive realm further down.

\subsection{Distributed Cognition}

During the so called cognitive turn, psychologists opened the former behaviorists' black box of the mind to conceptualize and investigate its workings as information processing activities, running on the quasi-computational infrastructure of the brain. These activities were originally described as information manipulation procedures, working like algorithmic rules on perceptual data and symbolic representations. This conception focusing on the inner workings of individual cognitive systems has been challenged and ecologically extended by subsequent approaches of situated and distributed cognition [30]. According to these theories, human cognition cannot be understood without its ubiquitous (self-)amplification and augmentation by cultural artifacts (like data carriers, calendars, compasses) or without its constant state of being "networked" with other processing units (social or anthropological perspective) [20]. In enculturated societies whole populations of cohabiting artefacts act as "things that make us smart" [38] as they unburden cognition (e.g. from strenuous exercises of imagination, computation or memorization) and enable the accomplishment of tasks far beyond unaided performance levels [30]. InfoVis interfaces - as an advanced species of interactive artefacts - thus extend and enhance cognitive systems into hybrid HCI ensembles, mediated by visual displays.

Fig. 1 shows the main components of such an extended cognitive system, enabling the visual analysis of complex objects of study (left) as external representations (ER) on visual displays (center) which augment corresponding internal representation (IR) in an analyst's working memory (more specifically on the visuo-spatial sketchpad). On these internally-externally coupled and distributed representations (DRs) reasoning and sensemaking operations can take place as visual-spatial manipulations. Results then can be stored as individual instances or generalized structures in long term memory (right).

\subsubsection{Data-Frame Theory of Sensemaking}

From a general sensemaking perspective visualizations or diagrams are just one possible way to organize and connect perceptual data with a cognitive frame [26] [21]. Other common frames which help analysts to integrate and interpret data from any observation are provided by concepts, stories, scripts, or various other sorts of images. Fig. 2 shows the basic data-frame symbiosis, where data provide raw perceptual stimuli, which are organized and interconnected by the higher-level cognitive structures of frames. According to their construction (which we will analyze as determined by syntactic rules), frames enable the meaningful connection of stimuli, and they also filter relevant from irrelevant data by steering attention and guiding perception.

From this perspective, individuals can acquire an extensive variety of cognitive frames as IRs, and constantly develop or learn new ones to ensure successful sensemaking and problem solving in ever-changing (task and data) environments. Following the insights of distributed cognition, they can also utilize external representations as devices to support their internal frame- and sensemaking activities. With regard to Fig. 1 (for an elaborated diagram see Fig. 4), the ER system (blue) thus augments the internal sensemaking system (green), and both components can interact in multiple ways.

\subsubsection{Dynamics between External and Internal Representations}

Across the distributed architecture of extended cognitive systems, mediated by InfoVis interfaces (Fig. 1), basic transaction processes play out in different combinations [31]:

i. If there is no prior knowledge or existing IR, internalization equals the construction of a new IR based on a given ER (i.e. learning). These processes require a bottom-up synthesis of visual micro-patterns into various forms of macro-level representations (see ch. 2.4). We will shift to the discussion of a new level of macro-syntactical connection methods further down.

ii. As acquired (and generalized) structures, existing IRs guide the

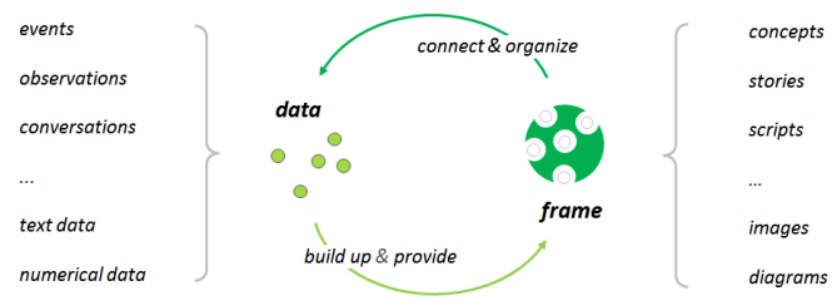

Fig. 2. The data-frame conception of sensemaking, which assumes a symbiotic relation between perceptual data (left) and cognitive frames (right), with the former building up the latter, while the latter connect and organize the former (adapted from [21]). 
processing and interpretation of newly appearing ERs [43]. We will focus on the construction and quality of macrorepresentational structures also because of evidence that only their coherent and consistent construction can effectively guide local sensemaking and visual reasoning [56].

iii. Existing IRs then can always be activated and simulated in working memory for visual reasoning operations, but can also be augmented by ERs to work in joint as coupled and distributed representations (DRs), which equals the standard constellation of an InfoVis-mediated HCI system. ${ }^{1}$

For such systems, numerous cognition-supporting effects have been described ([48], [7], [30], [31], [42]) and grouped into four functions [16]: Firstly, working memory is unburdened (from imagination, integration and memorization) by the external storage of detailed information on visual displays, which allows the corresponding internal representation to remain lean and lightweight. Secondly, the visual-spatial arrangement of information unburdens cognition from decoding abstract symbol sequences of language-like representations, to enable a more natural interpretation (e.g. of data items' relations or similarity via 'display proximity' [60]), and facilitates visual search and information integration. Thirdly, complex analytical operations can be offloaded to the swift workings of visual pattern recognition and preattentive processing [14]. Finally, strenuous symbol-based reasoning operations with abstract data could be offloaded to interactions with visual-spatial frames or models of the data. Users then can explore these visual models perceptually, and read off conclusions "without presupposing mental logics and formal rules" [31].

\subsection{Mental Models - Conceptual Origins}

Cognitive science offers a variety of concepts to address internal representations of external data in more depth. ${ }^{2}$ One specifically interesting approach comes with the theory of mental models: A mental model (MM) has been defined as an analogue, "small scale" representation of an external phenomenon [10]. Observations and explorations - e.g. in physical [56], as well as abstract, environments [22] - instruct "the creation and interpretation of an internal mental model" [53]. While exploring physical surroundings or information spaces - from caves to cities, and from libraries to complex datasets - users continuously build up models of observed objects, systems, or their environments and use these mental models to reason on them. The concept of a MM could be defined to be a specific type of "frame" - as defined by sensemaking theories ${ }^{3}$ - but with a stronger emphasis on a visual-spatial core structure, to which also other information modalities can connect. The practical relevance of mental models is their potential to "describe, explain and predict a system's purpose, form, function, and state" [15] and - in case of successful communication - to also act as shared mental model for collective understanding and decision making [54].

For more complex external objects or systems like machines or interfaces, two different understandings of mental models have developed, which lay their emphasis either on the structural or on the functional-behavioral aspects of a system [17]. While a structural model focuses on the spatial or topological arrangement of a system and its parts [22], a functional model represents its dynamic and

\footnotetext{
${ }^{1}$ Finally, if there is no existing ER yet, internally constructed IRs can also be externalized by creating novel visualization methods. Therefore also user-centred design recommends to let future users' existing IRs influence the design of novel ERs, so that the effective coupling of users' IRs and ERs is made more likely [42].

2 Prominent concepts include cognitive schemata, scripts, images, cognitive maps, prototypes, or frames [24].

3 "Sensemaking might mean the creation of a mental model. [...] Of all the psychological notions, this one seems closest to what people seem to mean today by sensemaking." [25].
}

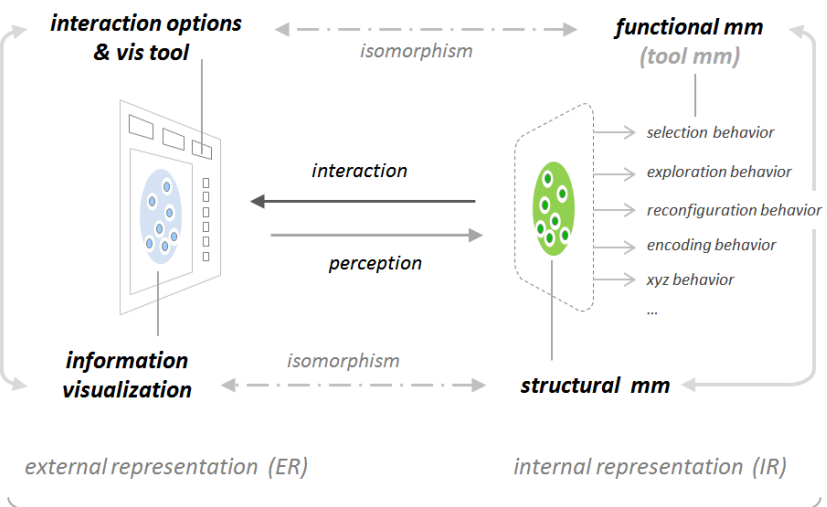

distributed representation (DR)

Fig. 3. Outline of a distributed representation, where the structure and behavior of an information visualization (left) is augmenting a mental model (right). Visual reasoning processes then equal the interaction with (i.e. exploration or manipulation of) this distributed, but coupled representations.

causal processes, including the conditional behavior of a system, like how something acts or how a device works when used in specific ways [37]. These conceptions provide a productively complementary perspective when it comes to understanding DRs and model-based reasoning (Fig.3).

When seeing data of a subject matter encoded into a visualization on a display, the analyst's structural model mirrors the "spatial, temporal or distributional relations between the data items" [31]. Complementing this perspective, the functional aspect of the model covers its possible dynamics, and how a visualization (system) works, especially via its interaction techniques. As Liu and Stasko summarize: A mental model is a functional analogue representation to an external interactive visualization system, which preserves structural and behavioral properties of external systems [31]. On the structural side, its focus is on the spatial layout of a visualization, but it also integrates other visual cues (color, hue, size, etc.) and also overlaid images and text [31]. With regard to the underlying data it can preserve schematic, semantic or item-specific information [31]. On the functional side, MMs include dynamic information about a structure's performance, and how it reacts to interactions.

Traditional descriptions of mental models furthermore agree in their non-veridic character: They commonly do not mirror ERs in an accurate or detailed manner, but they have to be good enough to ensure (task-specific) functionality or viability. Whether they are serving for descriptive, explanatory or predictive cognitive operations - IR mostly do so without even coming close to the complexity and full details of its corresponding ERs or subject matters. They can remain parsimonious, sketchy, and lightweight, and have to proof to be functional, viable or "runnable" for the achievement of certain tasks only [37]. This again is said to be possible only if a mental model is isomorphic to certain aspects of the ER, which again has to show isomorphic aspects with regard to the external phenomenon. ${ }^{4}$

\footnotetext{
${ }^{4}$ Held et al. delineate the required minimum fit of models and relevant external aspects as follows: "A perfect model is a model which is isomorphic to the whole relevant structure of the represented. If it is isomorphic to more or to less of the relevant structure (or even contains parts that are not shared by the represented), it is a bad model. A model containing too much will be more difficult to construct and to manipulate; therefore, it will make reasoning less effective. On the other hand, a model containing too little obviously will not be able to take over the functional role
} 


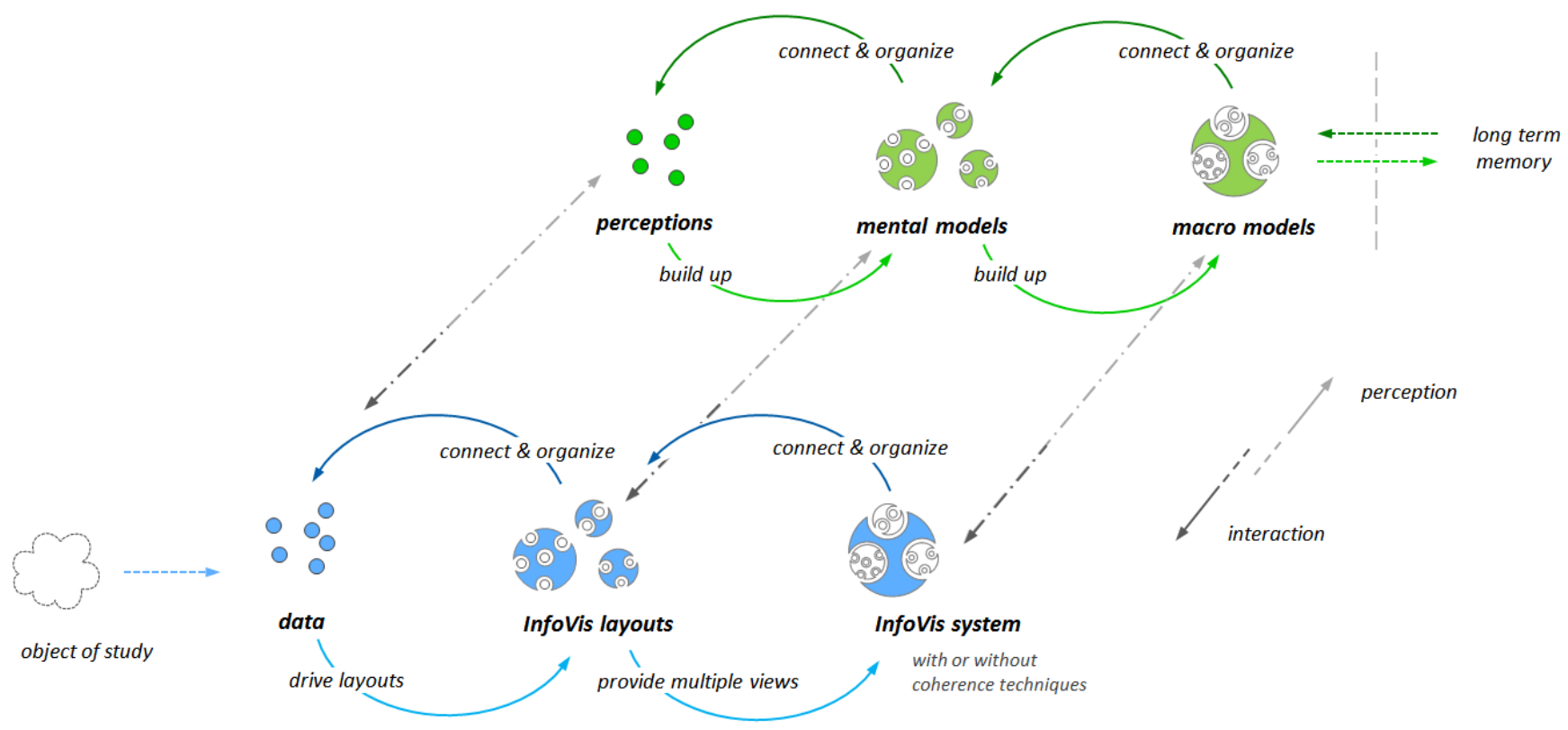

Fig. 4. Integrated depiction of a distributed macrocognitive system, with external representation components in blue, and internal representation components in green. Both layers contain basic data/frame cycles on the left hand side, which are coupled by perception and interactiondynamics (grey), with bright colors always denoting bottom-up effects and dark colors top-down effects in the system. This basic distributed sensemaking system then is extended with a macromodelling cycle (right hand side), which is can be supported by various coherence techniques of InfoVis systems with multiple views (ch. 3).

\subsection{Model Construction \& Visual Syntax}

Most of the work on mental models has been done with regard to subject matters, where a spatial layout is already given (e.g. geographic environments), and where the visual-spatial isomorphy between relevant aspects of external objects, ERs and IRs is easier to construct. The basic syntactical rule there is to preserve the spatial organization of selected data, which are already visually-spatially organized - similar to the methods of scientific visualization [49]. InfoVis systems, by contrast, translate abstract subject matters (commonly given as alphanumerical data) into visuo-spatial representations in a rule-based manner.

\subsubsection{Basic Syntax: Creating InfoVis Layouts Contained by Single Views}

The only general rule for proper ER design in the InfoVis domain is the mapping of selected relations or structures from an abstract (i.e. numerical or conceptual) data space to newly generated visualspatial relations and structures in the display space [16] [15]. As technically unlimited designs can solve this condition, many viable model types of diagrammatic ERs have been introduced, and their specific rules of construction are commonly referred to as InfoVis methods. These ensure - analogous to the grammar or syntax of a language, that elemental data items (visual marks and cues) can be drawn together and combined into meaningful composite representations like graphs, charts, or trees. If a shared understanding of the basic syntax (as rules of construction) is given, any visual encoding of data can be decoded, reconstructed and interpreted on the recipient's side.

adequately, since relevant pieces of information are missing" [18]. This pragmatist approach to the maximization of isomorphy for certain aspects between object, ER and IR, can productively be complemented by many other positions on the epistemological spectrum between realism and constructivism of visualizations [13].
As basic visual syntax (how to express meaning with diagrammatic pictures) they have to be learned (visual literacy), and have been culturally developed and codified relatively late - as compared to the syntax of classical and modern languages. ${ }^{5}$ Yet, when it comes to the question, how the basic or mid-level representations could be possibly organized into bigger pictures or global macro-models, a codified InfoVis macrosyntax is needed.

\subsubsection{Macrosyntax: Creating Coherent InfoVis Systems with Multiple Views}

Complex InfoVis systems frequently operate with multiple data projections or multiple views (MVs), which requires sensemaking on multiple levels. Fig. 4 draws together all distributed system components so far, to outline a decisive extension: Two interacting representational systems (InfoVis system in blue, cognitive system in green) are connected for the basic DR sensemaking cycle (with data/perceptions and InfoVis/MM coupling, left and center). If an InfoVis system includes multiple views (bottom right), a second sensemaking cycle is to be expected, dealing with the organization and integration of data into a macro-model or macro-frame (top, right hand side). For the first cycle the basic syntax is provided by various InfoVis methods, which determine how individual data points can be encoded, connected and organized. Similarly, to foster sensemaking with complex InfoVis systems, insights from multiple views (with their individual layouts) need to connected and organized. It can be assumed that the quality of the external means of

${ }^{5}$ For reference approaches to explicate the syntax or grammar of InfoVis methods and graphics from a general semiotic perspective, see Bertin [5] and Engelhardt [11]. While this work provides an indispensable basis for a more generic semiotic foundation of InfoVis, it stays practically restricted to covering basic syntax for individual views only - even if the recursive architecture of a "composite graphic object" allows to technically include visual macromodels too [11]). 
connection influence the quality of the coupled IR - on a meso as well as on a macro level.

\subsection{Mental Models vs. Cognitive Collages - On Differences of Coherence and Consistency}

When the basic syntactical rules of various InfoVis methods safeguard the external (and internal) synthesis of diagrams in individual views, the construction of next-level IRs (i.e. of "macromodels") has to integrate the different perspectives of multiple views (MVs). It can be assumed that such a comprehension process does not necessarily result in the creation of a coherent mental model, which covers the relevant aspects of complex subject matters in a visuo-spatially consistent way. Tversky [56] coined the term cognitive collages as a metaphor for the distorted mix-up of partial information, differing perspectives and reference points that characterize fragmentary IRs. Browsing information without macrostructural integration, can internally lead to "a very fragile network of knowledge and at worst to a quagmire of charming, but irrelevant pieces of information (and not knowledge)" [59]. In contrast to such inconsistent or poorly connected cognitive collages, mental models integrate different aspects and perspectives and "capture the categorical spatial relations among elements coherently, allowing perspective-taking, reorientation, and spatial inferences" [56].

We consider this introduction of a quality gradient for internal representations (from fragmented and distorted collages to coherent and consistent models) to be a distinction with possibly farreaching implications for the design and evaluation of InfoVis systems - and to be specifically relevant for macrocognitive reasoning tasks. ${ }^{6} \mathrm{We}$ hold that the safeguarding of reorientation, perspective-taking and spatial reasoning or inference operations is not only a relevant development goal for single methods development, but even more so for the design and evaluation of InfoVis systems with multiple views.

From a pragmatic user and task-analytical perspective it seems obvious, that oftentimes to work with vague cognitive collages can be good enough - or even more: the more easy and less effortful way of choice. But if future users of InfoVis systems should also be effectively supported with macrocognitive operations, then crucial cognitive construction efforts of macro-level syntheses should not be left to themselves. In fact we consider coherent "big-picture design" (across and in between) multiple views to be a high-level design task of its own. Amplification of macrocognition thus can shift from a rather arbitrary, casual, or non-existent mode of existence to a more system(at)ic and conscientious design practice, to specifically facilitate

- coherent information integration and consistent (structural and functional ) system level comprehension,

- reasoning and inference operations across different views, as well as the utilization of

- benefits from top-down effects of coherent macromodels on the local sensemaking operations with single views.

Yet if a coherent global synthesis is already challenging in face of different local perspectives where an overall spatial layout is given (and where also Tversky's reflections originated), it is an even more challenging task for InfoVis analysts: their macromodels do not only have to preserve certain structural and functional aspects of their local models, but also ensure their structural and/or functional interoperability, and bring them into a somehow consistent visuospatial macro-constellation.

\footnotetext{
${ }^{6}$ Tversky's suggested distinction has remained largely unnoticed so far, to which a conflation might have contributed, where the term "collage" has been identified with the MM concept, and read as a reference to its multi-layered nature, instead of being recognized as an antonym with regard to construction quality [31].
}

As such, the question of bigger picture design transforms into a basic investigation: Is the achievement of coherent and consistent mental macromodels for InfoVis possible at all? How can we support the construction of such visual marcocognitive superstructures? Are there known rules, practices, or methods as parts of a visual macrosyntax, which we can draw together? The next chapter collects and organizes supporting techniques to initially construct coherent models of abstract multidimensional data. Secondly, it assembles methods to integrate and connect multiple perspectives sequentially, and thirdly, it details what helps us to link multiple views synchronously or in parallel.

\section{Coherence Techniques as Binding Forces of a Visual Macro-SyntaX}

In the following we present methods and techniques which we expect to decidedly influence the macrocognitive construction of mental representations across multiple views. Building on the extended framework of model-based reasoning, we reviewed literature and tools to identify and systematize a first collection of coherence techniques for closer investigation. Overall, we delineate three different strategies:

- Methods for the initial construction of mental models (3.1) like advance organizers illustrate the conceptual relations between multiple views or data dimensions and thus support the initial setup of a globally consistent mental macro-model.

- Methods for sequential connection (3.2), like narrative visualization techniques or seamless transitions support the incremental elaboration or extension of an already existing mental model.

- Methods for parallel connection (3.3), like coordinated multiple views and coordinated interaction methods integrate local views in juxtaposition and thus provide synchronous visual cues for interconnection and integrated understanding.

\subsection{Methods to support the Initial Construction of Mental Macromodels}

Macromodels of InfoVis systems connect and organize insights and models from multiple views. To build up such a representational toplevel frame, various techniques can help to set up a structural or behavioral scaffold, which will influence later interaction with the system and will be enriched with more detailed data and insights during the ongoing interaction process.

Cognitive-syntactic principles: Based on their prior knowledge and the first impression of an InfoVis system users form a tentative mental model, which is further manipulated and elaborated in working memory. Thereby, new information can be connected to existing knowledge, it can be more easily processed and it is more closely tied into existing mental models. To assist and guide this initial construction process, we can actively manage users' first impressions by introducing the basic architecture as an advance organizer (3.1.1) or by providing transitions from known visualizations by onboarding techniques (3.1.2).

\subsubsection{Advance Organizers}

Advance organizers have been introduced as effective means to facilitate the construction of mental models [3]. In general an advance organizer is a pre-sketch of the information to be learned and allows to later integrate all information into this structure. InfoVis interfaces, which are extended with an advance organizer support the initial construction of a first mental model that can be enriched during further interaction.

Fig. 5 shows an example of an advance organizer depicting the basic structure of a multidimensional data set (left hand side), whose transformation and visual encoding into (multiple) single views could be traced by following the color-coded data dimension (center). The organization of these multiple views into a top-level 
representation could be provided by various layouts or architectures (right hand side), which can be further connected by various coherence techniques as elaborated below. We contend that any macro-shape or structure can act as a visuo-spatial scaffold. Numerous layouts of the local InfoVis spectrum are available for the organization of MVs on the macro-level too.

After providing initial construction support, advance organizers can be furthermore utilized to provide ongoing macrocognition support by using them also as a navigator to mediate between different views (see Fig. 5, bottom right, top left window). Navigators serve as constantly available overviews, which supports the ongoing cognitive integration and navigation between different perspectives or MVs by keeping a coordinated (over)view window present (see 3.3.1).

\subsubsection{Onboarding techniques and SciVis-InfoVis-transitions}

InfoVis layouts or diagrams are artificial and abstract(ed) images, whose syntactical rules have to be learned. Some of these rules of construction can also be introduced on the fly by various onboarding or transition techniques, which help to introduce the structure and workings of the InfoVis system (i.e. foreground), but also topic, context, and background knowledge. One interesting technique with regard to the origins of the mental models concept in spatial cognition research [56] are seamless transitions, which allow to trace the re-arrangement of familiar spatial and physical constellations into abstract InfoVis layouts [29] [9]. These technique help to transfer structural knowledge (and context) from existing mental models into unknown target constellations, and therefore act like sequential connection techniques (see 3.2), but with focus on the initial construction. The same transfer of knowledge (from spatial or SciVis layouts to InfoVis layouts - and vice versa) could also be implemented by parallel views (see 3.3), which keep both constellations present - so that both perspectives can mutually benefit from each other [49].

\subsection{Methods to Support the Sequential Interconnection of Multiple Views}

A second major principle for organizing multiple views is given by sequential presentation and connection over time. As such, temporal closeness provides the binding relation to connect different perspectives on the data. Practically, in its most basic version as an unmediated sequence, this technique utilizes an observer's memory, to hold transient perceptions and models present over time, and to make sense from (and store) their sequential relations. As such an unmediated information processing requires significant cognitive effort, various time-oriented techniques have been suggested to amplify sequential cognition and information integration.

Cognitive-syntactic principles: Results from different representation domains (for model-based text comprehension [22], for InfoVis [46]) provide evidence that it is cognitively more efficient to elaborate an existing mental model and transform it into a new arrangement, than to combine two different MMs. The unmediated presentation of two separate layouts can easily lead to two separate MMs, whereas sequential interconnections allow the adaptation and extension of an existing MM, resulting in less cognitive effort [22]. To support the sequential elaboration of one MM, coherence between multiple views can be indicated by perceptually traceable transformations in seamless layout transitions (3.2.1) and seamless canvas transitions (3.2.2), or by offering sequential guidance with narrative methods and coherence cues (3.2.3).

\subsubsection{Seamless Layout Transitions}

Seamless transitions produce coherence between different views by offering visually traceable change. This method uses various forms of morphing to visually translate from one spatialization and visualization method to another. By changing layouts incrementally as opposed to cutting abruptly - the spatial re-arrangement can be traced and the shifting of relevant, well-known elements can be followed smoothly. These techniques corresponds to the concept of preservation of the mental map [1], which aims at developing algorithms that keep the number of changing elements to a suitable minimum.

\subsubsection{Seamless Canvas Transitions}

Similar to layout transitions, seamless canvas transitions can help to visually trace and integrate the operating procedures of different views with specific regard to different dynamic visualization techniques [12]. Fig. 6 shows the traceable transitions between dynamic InfoVis methods as canvas movements, mediated by a generalized space-time cube [4]. Results of testing with users initially unfamiliar to such a system show that the informal learning appearing while watching in-between view "transitions could save hours to be spent with reading a manual otherwise" [52].

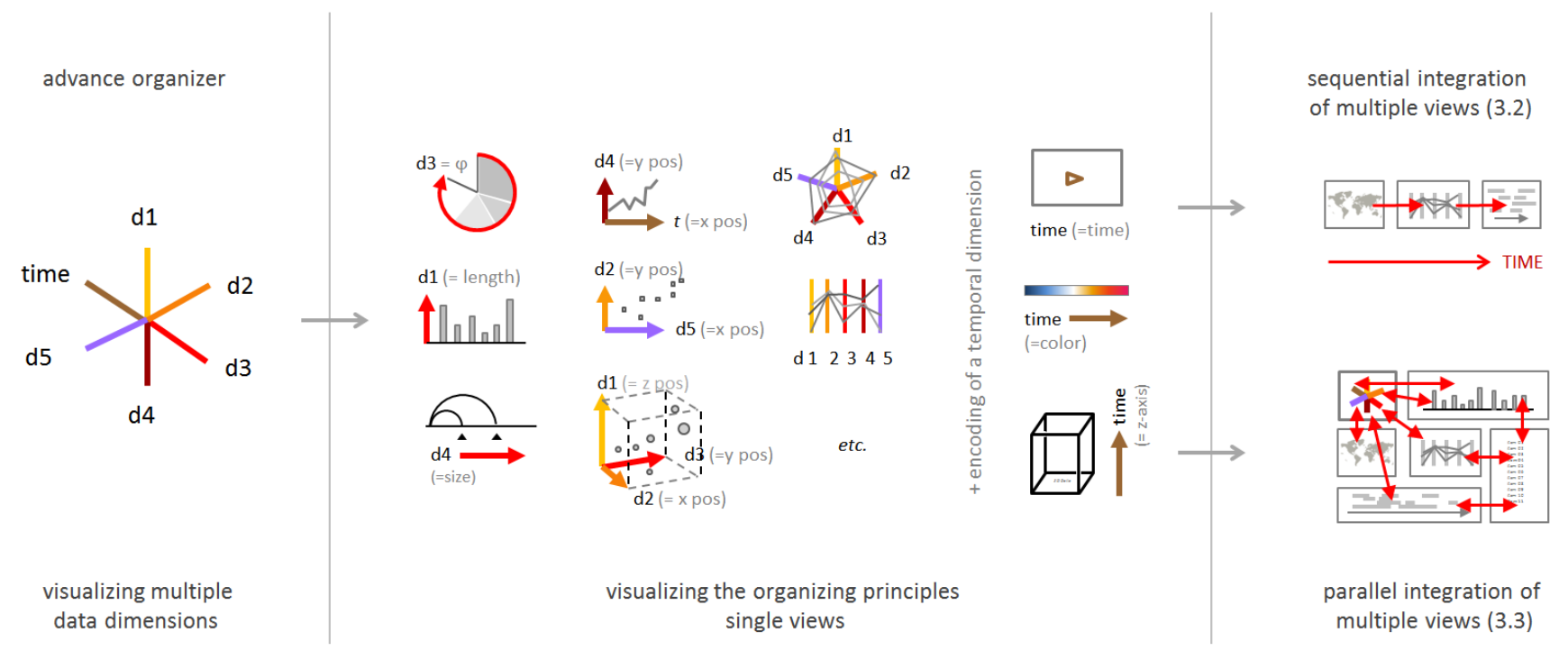

Fig. 5. Advance organizer (left) depicting the structure of a multidimensional data set, whose organization into various InfoVis layouts of single views (center) and higher-level representation (right) could be visually traced. 


\subsubsection{Incremental Elaboration of Existing MMs through Narrative User Guidance}

Multiple local views can also be connected into a global representation, by establishing and explicating chronological or causal connections between different views. Narrative guidance or storytelling has proven an effective means of communication and to "provide the connective tissue between facts to make them memorable" [27]. Stories have been defined as causally related chains of individual events or views and provide users with a clearly defined path through the data. One of the major design challenges for corresponding systems is the balancing of author-driven and readerdriven approaches [50]. Stories have been defined as causally related chains of individual events or views and provide users with a clearly defined path through the data. One of the major design challenges for corresponding systems is the balancing of author-driven and readerdriven approaches.

\subsection{Methods to Support the Parallel Interconnection of Multiple Views}

A third category of coherence techniques are methods to connect multiple views in parallel - either on the same or on neighboring displays. They make use of spatial relations (spatial-topological closeness / proximity) to synchronously connect different views.

Cognitive-syntactic principles: When confronted with multiple views in parallel, users have to compare the different views and connect the information from all views. If it is not possible to relate the views, they will build up multiple unconnected MMs, which have to be connected with huge effort later on [22]. To support the interconnection of multiple views and the construction of one coherent macro-model, different visual cues were suggested to semantically link individual views: consistent visual encoding of data in parallel juxtaposition (3.3.1), synchronous visual changes via coordinated interaction techniques (3.3.2), visual integration in a space-time-cube (3.3.3), or the provision of visual links between the views (3.3.4).

\subsubsection{Coordinated Multiple Views (CMVs)}

When multiple local views are given, one standard method for their global integration is their parallel juxtaposition, together with their interconnection by coordinated interaction techniques (see 3.3.2). By offering multiple views in parallel, complex datasets are brought into a global spatial constellation (or meta-frame) to be explored from complementary analytical perspectives by the means of synchronous contemplation [45]. We distinguish different types of multiple views: Mereologically diverse views (shared frame of reference or InfoVis method, but different data selections) and methodically diverse views (different InfoVis methods with same or different data selection), whose differing visual syntax exposes the strongest challenge for higher level integration.

In contrast to narrative techniques, the "connective tissue" has to be presented synchronously, and one basic technique to do so is self-consistent visual encoding of data and alignment of design choices for elements appearing across different views (like colors, labels, or directions of axes). Analysts then engage in juxtaposed sensemaking and potentially connect complementary information from diverse views. Further integration support is usually provided by coordinated interaction.

\subsubsection{Coordinated Interaction Methods}

Coordinated interaction methods subsequently allow the user-driven enrichment of between-view-connections with further coherence cues. The most common of these synchronous integration techniques are coordinated selecting and highlighting or linking and brushing, as well as synchronized panning, scrolling or zooming [40]. Coordinated interaction methods provide instant visual modifications of the same data elements in different views, which enable a perceptual integration of different views on demand. Such spatially unconnected but temporally synchronous changes allow relating and comparing selected visual marks or elements in different views, i.e. in different layout contexts or within different mereological data selections.

\subsubsection{Space-time Cube}

The space-time cube has been proposed as one specific method to serve as a device for conceptual integration, as an advance organizer and as a synchronous architecture integrating multiple spatial or time-oriented views. User studies confirmed that the detection of multidimensional patterns is easier than with multiple views [28]. The advantage of the space-time cube is that it provides a potent visual-conceptual device to merge many seemingly incoherent single views into one overview, which can serve as an integrative scaffold for a mental model on how to look on spatiotemporal data. For example, spatial and temporal perspectives could be mediated by a circular camera drive of 90 degree - from spatial to spatiotemporal to

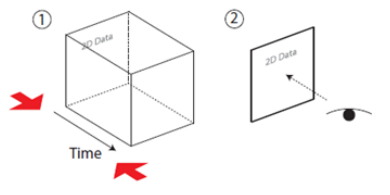

time flattening

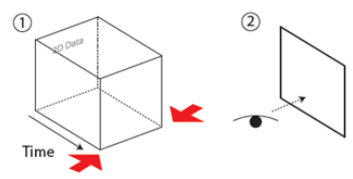

space flattening

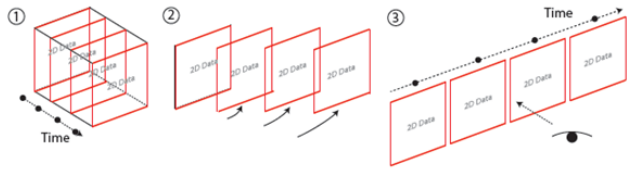

time juxtaposing

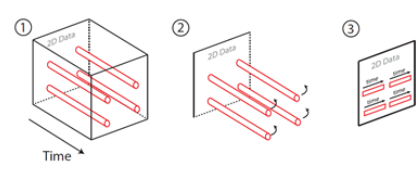

time drilling

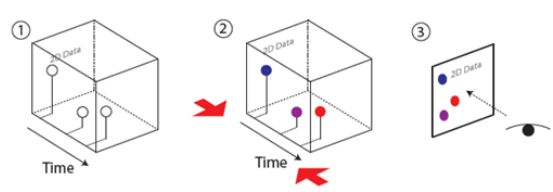

colored time flattening

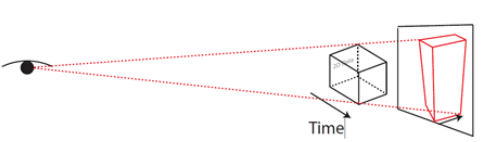

$3 D$ rendering

Fig. 5. Seamless Canvas transitions between the dynamic visualization methods of time flattening (top row from left), time juxtaposing, time drilling, space flattening (bottom row from left), colored time flattening, and 3D rendering of a conceptual space time cube by Bach [4], which also serves as a mediating transition framework. 
temporal - and vice versa. Various other spatial and temporal standard views, which usually produce different mental models, could be conceptually integrated with the space time cube - or coherently generated out of it (see Fig. 6).

\subsubsection{Visual Linking}

Another option to directly connect multiple views in parallel has been proposed by Collins and Carpendale [8]: As a method to explore relations between two different views, VisLink connects same data items on different canvases with visual ties or links. This method's principle builds on the parallel presentation and visual (meta-)connection of usually separated views, establishing a perceptual bridge between two systems or methods of local coherence. Canvases can be arranged in various spatial configurations, and bundles of inter-view-links provide insights into interrelationships across a diversity of diagrammatic regimes. This method could be imagined beyond two screens, but is expected to get visually complex soon.

Resúmé: We consider the assembled techniques so far to be a nonexhaustive collection, which aims for its extension and elaboration by a more systematic discussion and enrichment. As is rather obvious, this methods collection has to be complemented by evaluations of their expected macrocognitive effects. From a practical standpoint we expect this endeavor to also require novel evaluation techniques, which - given the spectrum of techniques equals an extensive research program.

\section{Towards an Evaluation Program of Coherence TECHNIQUES}

While the extension of the mental model approach to a macro-level of system design allowed a first assembly and discussion of multidimensional coherence techniques, we see the added value of this collection to also lie in its potential to guide empirical studies into the outlined higher level design challenge.

Empirical studies focusing on macrocognitive reasoning rather than artificial micro-level tasks are more similar to the everyday use of InfoVis tools. Thereby, the ecological validity of the empirical results increases. To guide macro-level evaluation we will first discuss evaluation scenarios, user and task dependency, and their different requirements for macrosyntax support. Then we will present different methods which could assist the evaluation of macrocognitive effects.

\subsection{Evaluation Scenarios}

The challenge to design coherent InfoVis systems on a macro-level and thus the need to adjust the implementation and evaluation of coherence techniques to the specifics of data, users, and tasks grows with increasing complexity of multidimensional datasets.

The required support depends on the user's expertise (with InfoVis syntax, but also with the topic and the datasets): As discussed in section 2.1.2, existing IR (including prior knowledge from long-term memory) influence the processing of and reasoning with ER. For example, it has been shown that experts draw more inferences from less coherent information systems, whereas nonexperts require more coherent systems to even build up a mental model [15]. Therefore, the implemented coherence techniques have to be adapted to the intended user group and users should be able to select the amount of support they need. This is especially important as some coherence techniques (e.g. seamless layout transitions) can slow down the user's exploration and reduce the user's freedom of action (e.g. authored systems). Therefore, we expect benefits from providing high support of coherence for the initial interaction with the InfoVis system (e.g., by an advance organizer or other onboarding methods), and from reducing the support later on to allow for more free exploration.
The question of supporting coherent representations should also be appropriate for the users' expected tasks: For many simple tasks, the rough orientation provided by cognitive collages may be a completely adequate solution, and a simple diagram may be a viable external representation. But when more global inferences and the generation of new multidimensional insights are involved, users require more coherent mental models to reason on a macro-level and consequently also more consistent InfoVis systems supporting such reasoning.

\subsection{Rethinking Evaluation Methods}

To evaluate the effects of coherence techniques on macrocognitive reasoning, methodologies are required to measure MMs and their overall quality and evaluation procedures have to be developed to single out the effects of individual coherence techniques on different macrocognitive reasoning processes.

\subsubsection{Assessing Mental Models}

To investigate how external representations influence the formation of coherent mental models in the InfoVis domain, specific evaluation techniques are required. In [34] we provide an overview of wellestablished methodologies from cognitive science to assess different quality indicators of mental models.

What defines the quality of a mental model? In this paper we focused on coherence, i.e. the consistency and interconnectedness of information elements [35]. In some contexts, it is important that the IR accurately mirrors structure and content of the ER. In other scenarios it might be important that the IR can be generalized and transferred to other datasets or tools. Also, Tversky [56] emphasizes the perspectivity of a mental model as a quality indicator in contrast to cognitive collages, allowing to flexibly inspect a $\mathrm{MM}$ from different viewpoints.

Depending on the most relevant quality indicator(s) for the evaluation scenario, different methodologies for the assessment of mental models should be selected or combined [33]. To assess the status quo of mental models, this can be accomplished with various techniques from cognitive science like interviews, rating [55] and sorting techniques [36] [41], but also more open structured methods like sketching techniques or concept maps. In contrast, most existing methods to evaluate InfoVis prototypes focus on the sensemaking process: Think-aloud techniques, the insight-method developed by Saraiya and North [47] or task-oriented procedures can be useful, but need some tuning, re-focusing, expansion or backing by additional methods to more accurately unveil users' construction of MMs and macrocognitive reasoning. For example, in [49] think-aloud protocols are analyzed to shed light on the users' knowledge construction processes: how they build up a mental model of the InfoVis tool and how it is related to their prior knowledge and mental model of the data.

An ideal evaluation combines process and status quo inquiries: Comparing the status quo of mental models before and after InfoVis tool use reveals the extent of knowledge gain. Process measures are better suited to gain an understanding of the construction of (macro)cognitive IRs and their ongoing manipulation and constant development within the distributed system. However, most of these measures are qualitative and therefore require higher evaluation effort than the comparison of status quo measures. Therefore, the selection of methodologies should not only depend on the quality indicators of interest and the evaluation scenario, but also on the available resources and the aim of the evaluation. Also, the first wave of research into macrocognitive support and processes should focus on qualitative process measures, to further extend our understanding how internal and external representations interact. Then, a second wave of research can experimentally test these assumptions with larger samples and more controlled status quo measures. 


\subsubsection{Evaluation Procedures}

In this paper we assembled a collection of diverse coherence techniques, which we expect to have effects on a macro-level (and thus should show effects in evaluation), but which also need to be tested more specifically. We consider this to be an extensive research endeavor, which will require coordinated evaluation effort by multiple InfoVis researchers. There is a need for systematic experimental research comparing different coherence techniques with each other, with combinations of techniques, and with InfoVis tools without macro-level support. And there is a need to include macrocognitive measures into these studies: Earlier, we delineated that coherence techniques can support and facilitate (1) coherent information integration and consistent (structural or functional) system level comprehension, (2) interoperable reasoning and inference operations across different views, as well as (3) the utilization of benefits from macro-level coherence via top-down effects on local sensemaking operations with single views. In the following we will outline three corresponding prototypical evaluation procedures:

To assess the first macrocognitive task level, we can evaluate the macro-level understanding of an InfoVis system in an experiment: Users will interact with the system either with or without coherence techniques. Afterwards, we compare the status quo of users' IR of the system using sorting or rating techniques. The results will allow conclusions about which system provides better support for the construction of macro-level system understanding. Further qualitative studies (e.g., with think-aloud techniques and interaction logs) can provide insights on the cognitive processes the system supports.

For the second level of macrocognitive tasks, we want to figure out how these techniques support reasoning across different views. For such experiments, a task-based approach seems to be a sensible choice: Users have to solve tasks which require integration of information from multiple views. Differences in time on task completion, error rates, and abortion rates provide insights into the supportive function of the used techniques. The most challenging part of such an experimental design is the development of tasks which require different levels of integration. Again, further qualitative studies can assist in the development of such tasks and provide insights how the system supports interoperable reasoning.

For the third level, we have to figure out the top-down effects of macro-level coherence on local reasoning. Here, a multi-level multi-method approach is necessary: We have to assess the coherence of the users' mental model (as described for the first level) and observe its influence on local task performance like success rates in identifying individual data points, or the enhanced recall of specific facts.

\section{Conclusion}

Currently, research on the cognitive side of interaction with InfoVis systems is often restricted to local task performance. Yet, the growing complexity and size of datasets requires more elaborate InfoVis tools implementing multiple views. This paper tried to draw a bigger picture: We extended the theoretical framework of distributed cognition and model-based reasoning into a macrocognition realm and discussed different approaches to macrolevel interface design. If sensemaking with complex data often equals the interconnected reasoning across multiple local views, developers of InfoVis interfaces can benefit from coherence techniques which allow to consistently connect and organize these perspectives on a global level and support users' macrocognitive reasoning processes. Implemented in well-conceived combinations, these techniques are expected to work like an infrastructure of cohesive forces beneath or between different views. Thus, they can amplify InfoVis users' macrocognitive syntheses by interconnecting multiple structural perspectives and functional model components within a more consistent bigger picture. Cognitive science research points to significant payoffs, which the deliberate implementation of such global coherence techniques can have on visual sensemaking: Users with more coherent mental models can better orient themselves in the information space, and benefit from enhanced inferring and perspective taking. Consequently, it seems crucial to more specifically direct research into questions how users can be provided with cues and techniques to coherently link information when designing InfoVis systems with multiple views.

What remains to be done is a systematic evaluation of the proposed coherence techniques to gain a better understanding of the underlying mechanisms, but also of their effects and effectiveness for different scenarios of use. To answer such macrocognitive research questions, to our knowledge no established evaluation procedures currently exist in InfoVis. Therefore, we presented methodologies for the assessment of macrocognitive $\mathrm{MM}$ and sketched possible evaluation designs for three macrocognitive tasks. In this paper, we were able to mention only some evaluation procedures, but several other empirical approaches can be imagined and many more research questions remain to be answered. We hope that these elaborations contribute to a new empirical focus on macrocognitive processes - also because coherence techniques usually are not at the forefront of evaluation and research attention

We consider the main contribution of these reflection to be:

a) The consolidation and extension of the theoretical framework of mental models and model-based reasoning to cover also macrocognitive tasks and methods in InfoVis: Currently no theoretical model for interaction with InfoVis exists [44]. The presented theoretical considerations emphasize the necessity of scalability for a theoretical framework - from local up to macrocognitive levels and will hopefully help to develop a shared theoretical framework.

b) The assembly and systematization of macro-syntactical coherence techniques to more consciously and pragmatically address this level of InfoVis system design: While not aiming at an exhaustive collection, we assembled and discussed a first sample of alleged connectivity-supporting system design methods which are expected to have effects on the users' generation of mental macro-models and their coherence by preserving and enabling as many(visual) synergies and interrelations of insights as possible.

c) The setup of a research agenda to guide empirical studies on the hypothesized effects of coherence techniques: While this last point could be easily underestimated, we consider it to be rather essential for the organization and initialization of a systematic and self-composed evaluation program with high complexity and on a large scale. Also, we attempt to shine a light on a multi-faceted evaluation challenge, which tends to vanish behind the salient research into the development and refinement of single view methods.

By presenting a macrosyntax to link multiple views in InfoVis systems, we also see a chance for a novel discussion that contributes to raising the interoperability standards of established InfoVis theories, terminologies and associated communities of practice. A better exchange could also be facilitated by the elaboration of local conceptual bridges, which still foster the global transactions between multiple theoretical frameworks and design paradigms. With this paper we hope to start a discussion on how to build a shared bigger picture of macrocognition in the context of InfoVis systems design.

\section{ACKNOWLEDGMENTS}

This work was supported in part by a IKT-Z grant from the Austrian Research Promotion Agency (FFG), Project No. 835937 and by a grant from the Austrian Science Fund (FWF), Project No. P28363. 


\section{RefERENCES}

[1] D. Archambault, and H. C. Purchase, "Mental map preservation helps user orientation in dynamic graphs," in Graph Drawing 2012, W. Didimo and M. Patrignani , Eds. Berlin Heidelberg: Springer, 2013, pp. 475-486.

[2] R. Arias-Hernandez, T. M. Green, \& B. Fisher, "From cognitive amplifiers to cognitive prostheses: Understandings of the material basis of cognition in visual analytics." Interdisciplinary science reviews, 37(1), pp. 4-18, 2012.

[3] D. P. Ausubel, "The use of advance organizers in the learning and retention of meaningful verbal material," J. of Educational Psychology, vol. 51, 1960, pp. 267-272.

[4] B. Bach, P. Dragicevic, D. Archambault, C. Hurter, and S. Carpendale, "A Descriptive Framework for Temporal Data Visualizations Based on Generalized Space-Time Cubes," in Computer Graphics Forum, 2016.

[5] J. Bertin, Semiology of Graphics: Diagrams, Networks, Maps,. ESRI Press, 2010.

[6] S. Card, J. Mackinlay, and B. Shneiderman, Readings in information visualization: Using vision to think. San Francisco: Morgan Kaufmann, 1999.

[7] S. Card, "Information visualization", in The human-computer interaction handbook: Fundamentals, evolving technologies, and emerging applications, A. Sears and J.A. Jacko, Eds. New York: Lawrence Erlbaum, 2008, pp. 509-544.

[8] C. Collins and S. Carpendale, "VisLink: Revealing relationships amongst visualizations," IEEE Transactions on Visualization and Computer Graphics, vol. 13, no. 6, pp. 1192-1199, 2007.

[9] O. Cornec and R. Vuillemo,: "The Globe of Economic Complexity", http://globe.cid.harvard.edu/

[10] K. Craik. The Nature of Explanation. Cambridge University Press, Cambridge, 1943

[11] J. v. Engelhardt, The language of graphics: A framework for the analysis of syntax and meaning in maps, charts and diagrams. Yuri Engelhardt. (2002).

[12] P. Federico, W. Aigner, S. Miksch, F. Windhager, and L. Zenk, "A visual analytics approach to dynamic social networks," in Proceedings of the 11th International Conference on Knowledge Management and Knowledge Technologies, New York, NY, USA, 2011, p. 47:1-47:8

[13] P. I. A. Fernández and M. Buchroithner, Paradigms in Cartography: An Epistemological Review of the 20th and 21st Centuries. Springer Science \& Business Media, 2013.

[14] C.G. Healey, K.S. Booth, J.T. Enns, J.T. (1996). High-speed visual estimation using preattentive processing. ACM Trans. Comput . Hum. Interact. (TOCHI), 3(2), pp. 107-135, 1996.

[15] M. Hegarty. Diagrams in the mind and in the world: Relations between internal and external visualizations. Lecture notes in computer science, pages 1-13, 2004.

[16] M. Hegarty, "The cognitive science of visual-spatial displays: Implications for design,” Topics in Cognitive Sci., vol. 3, 2011, pp. 446-474.

[17] J. Heiser and B. Tversky, "Arrows in comprehending and producing mechanical diagrams," Cognitive science, vol. 30, no. 3, pp. 581-592, 2006.

[18] C. Held, M. Gottfried, G. V. Vosgerau, and M. Knauff, Mental models and the mind: current developments in cognitive psychology, neuroscience and philosophy of mind, vol. 138. Elsevier, 2006.

[19] S.C. Hirtle, "Ordered trees: A structure for the mental representation of information." Advances in Classification Research Online, Vol 1., pp. 79-86, 1990.

[20] E. Hutchins, "Cognition in the Wild," Cambridge: The MIT Press, 1995.

[21] R. Hutton, G. Klein, and S. Wiggins, "Designing for sensemaking: A macrocognitive approach," in Sensemaking Workshop, CHI, 2008, vol. 8.

[22] P. N. Johnson-Laird, "Mental models in cognitive science," Cognitive Sci., vol. 4, no. 1, 1980, pp. 71-115.
[23] N. Jones, H. Ross, T. Lynam, P. Perez, and A. Leitch, A, "Mental models: An interdisciplinary synthesis of theory and methods," Ecology \& Soc., vol. 16, no. 1, 2011, p. 46.

[24] G. Klein and R.R. Hoffman. Macrocognition, mental models, and cognitive task analysis methodology. In Naturalistic Decision Making and Macrocognition, J. M. Schraagen, L. G. Militello, T. Ormerod, and R. Lipshitz, Eds., 2008, pp. 57-81.

[25] G. Klein, B. Moon, and R. R. Hoffman, "Making sense of sensemaking 1: Alternative Perspectives," IEEE intelligent systems, vol. 21, no. 4, pp. 70-73, 2006.

[26] G. Klein, J. Phillips, E. Rall, and D. Peluso, "A Data-frame theory of sensemaking," in Expertise Out of Context: Proceedings of the Sixth International Conference on Naturalistic Decision Making, R. R. Hoffman, Ed. New York, NY: Lawrence Erlbaum Assoc Inc, 2007, pp $113-155$.

[27] R. Kosara, and J. Mackinlay, "Storytelling: The next step for visualization," Comput., vol. 46, no. 5, 2013, pp. 44-50.

[28] P. O. Kristensson et al., "An evaluation of space time cube representation of spatiotemporal patterns," Visualization and Computer Graphics, IEEE Transactions on, vol. 15, no. 4, pp. 696-702, 2009.

[29] M. Lambrechts, "Rock'n'Poll": http://rocknpoll.graphics/

[30] Z. Liu, N. J. Nersessian, and J. T. Stasko. Distributed cognition as a theoretical framework for information visualization. IEEE Transactions on Visualization and Computer Graphics, 14(6):1173-1180, Dec. 2008

[31] Z. Liu, and J. T. Stasko, "Mental models, visual reasoning and interaction in information visualization: A top-down perspective," IEEE Transactions on Visualization \& Comput. Graph., vol. 16, 2010, pp. 999-1008.

[32] T. Lynam et al., "Waypoints on a journey of discovery: mental models in human-environment interactions," Ecology and Society, vol. 17, no. $3,2012$.

[33] J. P. Magliano, J. Miller, and R. A. Zwaan, "Indexing space and time in film understanding," Applied Cognitive Psychology, vol. 15, no. 5, pp 533-545, 2001.

[34] E. Mayr, G. Schreder, M. Smuc, and F. Windhager. 2016. Looking at the Representations in Our Mind: Measuring Mental Models of Information Visualizations. Proceedings of the Beyond Time and Errors on Novel Evaluation Methods for Visualization, ACM, 96-103.

[35] D. S. McNamara, E. Kintsch, N. B. Songer, and W. Kintsch, "Are good texts always better? Interactions of text coherence, background knowledge, and levels of understanding in learning from text," Cognition and instruction, vol. 14, no. 1, 1996, pp. 1-43.

[36] L. A. McNamara and N. Orlando-Gay, "Reading, sorting, marking, shuffling". BELIV Workshop 2012, 2012.

[37] D. A. Norman. "Some observations on mental models." Mental models, pages 7-14, 1983.

[38] D. A. Norman, "Things that make us smart: Defending human attributes in the age of the machine." Basic Books, 1993.

[39] C. North. "Toward measuring visualization insight." IEEE Comput Graph Appl 2006; 26(3): 6-9.

[40] C. North, and B. Shneiderman, "Snap-together visualization: can users construct and operate coordinated visualizations?," Int. J. of HumanComput. Stud., vol. 53, no. 5, 2000, 715-739.

[41] J. R. Olson and H. H. Rueter, "Extracting expertise from experts: Methods for knowledge acquisition.” Expert Systems, Vol. 4, No. 3, pp. 152-168, 1987.

[42] R. E. Patterson et al., "A human cognition framework for information visualization," Computers \& Graphics, vol. 42, pp. 42-58, 2014.

[43] P. Pirolli and S. Card, "The sensemaking process and leverage points for analyst technology as identified through cognitive task analysis," in Proceedings of international conference on intelligence analysis, 2005, vol. 5, pp. 2-4.

[44] M. Pohl, M. Smuc, and E. Mayr, "The user puzzle - Explaining the interaction with Visual Analytics systems," IEEE Trans. on Visualization and Comput. Graph., vol. 18, 2012, pp. 2908-2916.

[45] J. C. Roberts, "State of the art: Coordinated \& multiple views in exploratory visualization," in Coordinated and Multiple Views in Exploratory Visualization, 2007. CMV'07. Fifth International Conference on, 2007, pp. 61-71. 
[46] P. Ruchikachorn, and K. Mueller, "Learning Visualizations by Analogy: Promoting Visual Literacy through Visualization Morphing," IEEE Trans. on Visualization and Comput. Graph., vol . 21, no. 9, 2015, pp. 1028-1044.

[47] P. Saraiya, C. North, und K. Duca, "An Insight-Based Methodology for Evaluating Bioinformatics Visualizations", IEEE Trans. on Visualization and Comput. Graph, Vol. 11, No. 4, p. 1, 2005.

[48] M. Scaife and Y. Rogers. External cognition: how do graphical representations work? Int. J. Human-Computer Studies, 45(2):185-213, 1996.

[49] M. Sedlmair, K. Ruhland, F. Hennecke, A. Butz, S. Bioletti, and C. O'Sullivan, "Towards the big picture: Enriching $3 \mathrm{~d}$ models with information visualisation and vice versa," in Smart Graphics, 2009, pp. 27-39.

[50] E. Segel, and J. Heer, "Narrative visualization: Telling stories with data," IEEE Transs on Visualization and Comput. Graph., vol. 16, no. 6, 2010, pp. 1139-1148.

[51] M. Smuc, E. Mayr, T. Lammarsch, W. Aigner, S. Miksch, and J. Gärtner, "To score or not to score? Tripling insights for participatory design,” IEEE Comput. Graph. Appl., vol. 29, no. 3, 2009, pp. 29-38.

[52] M. Smuc, P. Federico, F. Windhager, W. Aigner, L. Zenk, and S. Miksch, "How do you connect moving dots? Insights from user studies on Dynamic Network Visualizations," in Human Centric Visualization: Theories, Methodologies and Case Studies, T. Huang, Ed. New York, NY, USA: Springer, 2012, pp. 623-650
[53] R. Spence, “A framework for navigation,” Int. J. Human-Comput. Stud., vol. 51, 1999, pp. 919-945.

[54] R. J. Stout, J. A. Cannon-Bowers, E. Salas, and D. M. Milanovich, "Planning, shared mental models, and coordinated performance: An empirical link is established," Human Factors: The Journal of the Human Factors and Ergonomics Society, vol. 41, no. 1, pp. 61-71, 1999.

[55] F. B. Tan and M. G. Hunter "The Repertory Grid Technique: A Method for the Study of Cognition in Information Systems," MIS Quarterly, Vol. 26, pp. 1, 2002.

[56] B. Tversky, "Cognitive maps, cognitive collages, and spatial mental models," in Spatial Information Theory. A Theoretical Basis for GIS, A. U. Frank and I. Campari, Eds. Berlin: Springer, 1993, pp. 14-24.

[57] B. Tversky, "Visualizing thought," Topics in Cognitive Sci., vol. 3, 2011, pp. 499-535.

[58] T. A. van Dijk, and W. Kintsch, Strategies of discourse comprehension. New York: Academic Press, 1983.

[59] J.J. van Merriënboer, and P. A. Kirschner, P. A., Ten steps to complex learning: A systematic approach to four-component instructional design, 2nd ed. New York: Routledge, 2012.

[60] C. D. Wickens, \& M. Carswell, "The proximity compatibility principle: Its psychological foundation and relevance to display design," Human Factors, 37, pp. 473-494, 1995. 